Case report

\title{
Congenital acute myeloid leukemia in Down syndrome
}

\author{
Elena Andreea Moaleș ${ }^{1}$, Mirabela Smaranda Subotnicu ${ }^{*}, 2$, Denisa Boroș ${ }^{2}$, Anca \\ Ivanov $^{2,3}$, Adriana Mocanu ${ }^{2,3}$, Magdalena Stârcea ${ }^{2,3}$, Ingrith Miron ${ }^{2,4}$ \\ ${ }^{1}$ Department of Pneumology, Clinical Hospital of Pneumology, lași, Romania, "'Grigore T. Popa" \\ University of Medicine and Pharmacy, Iași, Romania, ${ }^{3}$ Department of Pediatrics, Emergency Hospital \\ for Children "Sf. Maria" Iași, Romania, ${ }^{4}$ Department of Pediatric Hematology-Oncology, Emergency \\ Hospital for Children "Sf. Maria", Iași, Romania
}

\begin{abstract}
Congenital leukemia is an uncommon myeloproliferative disorder usually diagnosed in the first month of life. Newborns with Down syndrome have an increased susceptibility to acute megakaryoblastic leukemia. We report a case of a newborn with Down syndrome diagnosed with acute myeloid leukemia. The diagnosis was established by clinical and hematological work-up.
\end{abstract}

Keywords: congenital leukemia; newborn; Down syndrome

\section{Introduction}

Congenital leukemia (CL) is an uncommon myeloproliferative disorder of the newborn, with an incidence of $4.3-8.6$ per million live births [1]. Down syndrome (DS) is the most common chromosomal disorder, affecting 1 in every 700 newborns. Hematological abnormalities in a newborn with DS include a variety of benign and malignant disorders. Patients with DS are found to have an increased incidence of both acute lymphoblastic leukemia (ALL) and acute myeloid leukemia (AML) - most frequent AMLM7 type [2, 3].

Received: March 2018; Accepted after review: June 2018; Published: June 2018.

${ }^{*}$ Corresponding author: Mirabela Smaranda Subotnicu, "Grigore T. Popa" University of Medicine and Pharmacy, 16 Universitatii Street, lasi, Romania.

Email: subotnicu mirabela@yahoo.com

\section{Case report}

A term female infant was born by vaginal delivery with normal birth weight, body length and APGAR score, from a 42-year-old mother with 13 previous pregnancies resulting in 3 miscarriages and 10 live births. The mother had no history of antenatal medical illness nor of exposure to smoking, drinking and other drugs. At birth, general and systemic examination revealed a round face, single palmar crease, left precordial systolic murmur. Two hours after birth a deterioration of the general condition occurred, with generalized hypotonia, cyanosis, poor feeding. The blood count revealed white blood cell count of $35.6^{*} 10^{3} / \mu \mathrm{L}$ with $20.6^{*} 10^{3} / \mu \mathrm{L}, \quad 57.9 \%$ monocytes, normal neutrophils, lymphocytes and eosinophils count, hemoglobin levels of $19.1 \mathrm{~g} / \mathrm{dl}$ and $27^{*} 10^{3} / \mu \mathrm{L}$ platelets count. The acute phase reactants were negative. Because she maintained the altered general condition and the platelets ranged between $17-18 * 10^{3} / \mu \mathrm{L}$, on the 8th day after birth she was referred to our unit for proper diagnosis and treatment. Physical examination showed a 
phenotype suggestive for Down syndrome, later confirmed by karyotyping $(47, X X+21)$. She was lethargic, tachypneic and a systolic heart murmur was observed. The liver was 2 $\mathrm{cm}$ below the right costal margin, along with a slight enlargement of the spleen. The laboratory tests on the first day of admission in our unit revealed a white blood count of $15.8^{*} 10^{3} / \mu \mathrm{L}$, with an abnormal monocyte count (increased absolute and percentile count: $5.66^{*} 10^{3} / \mu \mathrm{L}$, respectively $35.5 \%$ ), normal absolute neutrophil count $\left(5.53^{*} 10^{3} / \mu \mathrm{L}\right)$, a hemoglobin level of $15.9 \mathrm{~g} / \mathrm{dl}$ and severe thrombocytopenia $\left(15^{*} 10^{3} / \mu \mathrm{L}\right)$. The biochemical parameters including electrolytes, uric acid, creatinine, bilirubin, liver enzymes were normal. The serum lactate dehydrogenase was raised. The bacterial culture work-up and titers of antibodies against toxoplasmosis, cytomegalovirus, Epstein Barr virus, hepatitis C, HIV were negative. The peripheral blood smear presented atypical cells. The bone marrow aspiration showed hemodiluted aspirate with blast cells. Immunophenotyping revealed $23 \%$ blast cells, positive for megakaryocytic markers (CD42b, CD41, CD61), myeloid markers (CD33), progenitor cell markers (CD117, CD34) and T cell marker - CD7 positive. MPO and HLA/DR were negative. The mutational status of $A M L-$ ETO, PML-RARQ, FLT3 and NPM1 fusion genes came out absent. The positive diagnosis was acute megakaryoblastic leukemia (AMKL).

The echocardiography found a patent foramen ovale. The infant underwent chemotherapy according to the Down syndrome-specific AML chemotherapy protocol, consisting in four cycles of treatment: the first two cycles (induction phase) included combinations of cytarabine and liposomal daunorubicin and the last two cycles (consolidation phase): etoposide, cytarabine and mitoxantrone. Our patient aquired clinical and hematological remission without serious adverse events.

\section{Discussion}

Congenital leukemia (CL) is an extremely rare pathology, with a high mortality rate. This rare pathology, with a high mortality rate. This condition applies to children in their first month of life [4]. In this age group, acute myeloblastic leukemia is more common than acute lymphoblastic leukemia, and among the subtypes of AML, subtypes M5 and M4 account for approximately $50 \%$ of congenital leukemia; the M7 subtype is little involved [3, $5,6]$. Acute megakaryoblastic leukemia (AMKL) is a subtype of acute myeloid leukemia, a rare heterogenous malignancy in adults and more frequent in children, characterized by abnormal megakaryoblasts that are immunophenotypically positive for CD41, CD42b and CD61.

Extensive myelofibrosis in the bone marrow has also been reported [7-9]. Congenital leukemia is associated with certain genetic pathological entities such as Down syndrome, Turner syndrome or Klippel-Feil syndrome [10]. Trisomy 21 was detected in our patient.

Children with DS are at high risk of developing hematological abnormalities such as neutropenia, thrombocytopenia, myelodysplasia, acute myeloid leukemia, acute lymphoid leukemia [11]. In young children with Down syndrome, there is a 46 to 83-fold increased risk of acute myeloblastic leukemia. AMKL is estimated to be 400 times than other children from general population and accounting for $50 \%$ of all cases of AML associated with DS. Newborns with DS have a 20 -fold increased risk of developing acute leukemia [12-14].

The GATA-1 gene encodes the essential hematopoietic transcription factor. Acquired mutations in the GATA-1 gene have been found in blast cells in DS with myeloid leukemia [15]. The GATA1 mutation could not be performed in our patient. The most common clinical signs encountered immediately postnatal in most newborns are respiratory distress, possibly due to secondary leukemic infiltration, hepato-splenomegaly, petechiae, and in exceptional cases leukemia, cutis [16]. Signs of respiratory distress lethargy, hepatosplenomegaly were noted in our patient immediately after birth, with no skin manifestations. The laboratory workup revealed myeloid blasts on both blood smear and bone marrow, confirmed by flowcytometry. 
The diagnosis criteria of $\mathrm{CL}$ according to Bresters et al. are: presentation in the first month of life, proliferation of immature myeloid, lymphoid or erythroid cells, infiltration of these cells into non-haematopoiectic tissues and absence of other diseases that may explain the proliferation [5]. In $4-10 \%$ of cases, Down syndrome associates transient myeloproliferative disease (TMD), this being one of the most important differential diagnoses. TMD may mimic acute leukemia and presents hepatic infiltration, pancytopenia, blast cells in bone marrow and spontaneous remission within three to seven months of onset without any chemotherapic treatment [3, $5,17]$. The differential diagnosis of $\mathrm{CL}$ also includes intra-uterine infections and sepsis, which in our patient were excluded by viral serology and negative peripheral cultures [3].

The treatment of congenital leukemia means exposure of the newborn to toxic chemotherapeutic agents. Children with DS have an increased risk of secondary cardiac toxicity to anthracycline due to the existing cardiac malformations in this disease [18]. The ultrasound aiming to reveal patent foramen ovale and cardiac follow-up is especially indicated in this population. The prognosis of $\mathrm{CL}$ is poor, with a surviving rate of $23 \%$ at 2 years [5]. AMKL associated with Down syndrome (DS-AMKL) has a superior outcome compared with the sporadic form, with longterm survival $[3,19]$. The follow-up period for our patient was 1 year and 3 months and she

\section{References}

1. Bajwa RP, Skinner R, Windebank KP, Reid MM. Demographic study of leukaemia presenting within the first three months of life in the northern health region of England. J Clin Pathol 2004; 57:186-188.

2. Prakash KP, Rau ATK, Bhat ST, Rau AR. Congenital Leukemia - A Diagnostic Dilemma. Indian J Med Paediatr Oncol 2008; 29(4):4143.

3. Roy A, Roberts I, Norton A et al. Acute megakaryoblastic leukaemia (AMKL) and transient myeloproliferative disorder (TMD) in Down syndrome: a multi-step model of myeloid leukaemogenesis. Br J Haematol 2009; 147:312. is still in complete remission. Our case is similar with the literature data in which children with DS-AMKL present a better treatment response and survival rate compared to children with sporadic AMLK. In order to assess disease evolution, periodical clinical and hematological work-up (CBC and bone marrow aspiration) need to be performed.

\section{Conclusion}

The prognosis of congenital leukemia depends on the immunocytological form and the tolerance of the newborn to chemotherapy. Recent studies suggest a superior outcome in children with Down syndrome and AML compared to children with sporadic AMLK. For our patient a prolonged follow-up period is needed.

\section{Conflict of interest}

The authors have no conflict of interest to declare.

\section{Patient consent}

Written informed consent was obtained from the patient's parents for publication of this case report and accompanying images. A copy of the written consent is available for review by the Editor-in-Chief of this journal.
4. Sande JE, Arceci RJ, Lampkin BC. Congenital and neonatal leukemia. Semin Perinatol 1999;23(4):274-285.

5. Bresters D, Reus ACW, Veerman AJP, et al. Congenital leukaemia: the Dutch experience and review of the literature, $\mathrm{Br} J$ Haematol 2002; 117(3):513-524.

6. Isaacs Jr. H. Fetal and neonatal leukemia. J Pediatr Hematol Oncol 2003; 25(5):348-361.

7. Gruber TA, Downing JR. The biology of pediatric acute megakaryoblastic leukemia. Blood 2015; 126(8):943-949.

8. De Rooij JDE, Branstetter $\mathrm{C}, \mathrm{Ma} \mathrm{J}$, et al. Pediatric Non-Down Syndrome Acute Megakaryoblastic Leukemia is Characterized 
by Distinct Genomic Subsets with Varying Outcomes. Nat genet 2017; 49(3):451-456.

9. Schweitzer J, Zimmermann M, Rasche M, et al. Improved outcome of pediatric patients with acute megakaryoblastic leukemia in the AMLBFM 04 trial. Ann Hematol 2015; 94:13271336.

10. Warrier RP, Emami A, Ravindranath $Y$ et al. Perinatal Leukemia: The surface antigens, cytochemistry and cytogenetic features. Indian Pediatr 1983; 20:325-333.

11. Henry E, Walker D, Wiedmeier SE, Christensen RD. Hematological abnormalities during the first week of life among neonates with Down syndrome: data from a multihospital healthcare system. Am J Med Genet 2007; 143:42-50.

12. Lange $B$. The management of neoplastic disorders of haematopoiesis in children with Down's syndrome. $\mathrm{Br} J$ Haematol 2000; 110:512-524.

13. Mathur NB, Joshi $N$, Singh $T$, Singh $M$. Congenital acute megakaryocytic leukemia. Indian J Med Paediatr Oncol 2011; 32(3):165167.

14. Fatima Shirly AG, Darlington D. A rare case of congenital leukemia: acute myeloblastic leukemia in a neonate with Down syndrome. Int J Contemp Pediatr 2016; 3(1):288-290.

15. Wechsler J, Greene M, McDevitt MA, et al. Acquired mutations in GATA1 in the megakaryoblastic leukemia of Down syndrome. Nat Genet 2002; 32:148-152

16. Campwell WA, Storlazzi E, Vintizileos AM. Fetal neonatal leukemia. Arch Dis Child 1962; 37:93-98.

17. Gamis AS, Alonzo TA, Gerbing RB, et al. Natural history of transient myeloproliferative disorder clinically diagnosed in Down syndrome neonates: a report from the Children's Oncology Group Study A2971. Blood 2011; 26:6752-6759.

18. O'Brien M, Taub J, Stine $K$, et al. Excessive cardiotxocity despite excellent leukemia-free survival for pediatric patients with Down syndrome and acute myeloid leukemia: results from POG (Pediatric Oncology Group) protocol 9421. Blood 2006; 108:168a-169a.

19. Rao A, Hills RK, Stiller C, et al. Treatment for myeloid leukaemia of Down syndrome: population-based experience in the UK and results from the Medical Research Council AML 10 and AML 12 trials. $\mathrm{Br} J$ Haematol 2006; 132:576-583. 